\title{
Perfis Enzimáticos de Genótipos de Caesalpinia ferrea var. leyostachia e Cassia grandis
}

\author{
Allívia Rouse Ferreira dos Santos ${ }^{1}$, Erica Moraes de Souza ${ }^{2}$, Renata Silva-Mann ${ }^{3}$, \\ Robério Anastácio Ferreira ${ }^{3}$, Ana Veruska Cruz da Silva ${ }^{4}$ \\ ${ }^{1}$ Universidade de Santiago de Compostela - USC \\ ${ }^{2}$ Universidade Federal de Lavras - UFLA \\ ${ }^{3}$ Universidade Federal de Sergipe - UFS \\ ${ }^{4}$ Embrapa Tabuleiros Costeiros
}

\begin{abstract}
RESUMO
A Caesalpinia ferrea var. leyostachia (pau-ferro) e Cassia grandis (canafístula) apresentam importância para obtenção de mudas para recuperação de áreas degradadas, porém pouco se tem na literatura sobre estas espécies. Objetivando-se identificar diferenças em perfis enzimáticos destes indivíduos foi realizado o presente trabalho. Realizou-se extração de enzimas Álcool Desidrogenase, Esterase, Glutamato Oxaloacetato Transaminase, Peroxidase e Glucose Desidrogenase. Em apenas três dos sistemas observou-se atividade, sendo a Esterase a que apresentou maior número de bandas. Notaram-se perfis diferenciados entre os genótipos de canafístula e entre os genótipos de pau-ferro avaliados, permitindo-se inferir sobre a diversidade existente entre os indivíduos.
\end{abstract}

Palavras-chave: variabilidade genética, sementes, isoenzimas.

\section{Enzymatic Profile of Caesalpinia ferrea var. Leyostachia and Cassia grandis}

\begin{abstract}
Caesalpinia ferrea var. Leyostachia and Cassia grandis are important species for obtaining and propagating seedlings for reforestation in degraded areas, but rare is the information about those species in the literature. This study was carried out to estimate the genetic diversity between individuals of $C$. ferrea and between individuals of C. grandis. The enzymes used were the following: Alcohol Dehydrogenase, Esterase, Glutamate Oxaloacetate Transaminase, Peroxidase and Glucose Dehydrogenase. Only three of the systems presented activities and the Esterase system presented a higher number of bands. Differentiated patterns between C. ferrea individuals and between C. grandis individuals were observed, thus, permitting to infer that there is genetic diversity of individuals.
\end{abstract}

Keywords: genetic variability, seed, isoenzymes. 


\section{INTRODUÇÃO}

O Estado de Sergipe sofre com as transformações causadas pela destruição dos recursos naturais relacionadas com a mata ciliar e florestas naturais, restando destes, faixas isoladas e distantes, contribuindo para a homogeneidade da natureza. Sendo assim, é necessário um estudo da diversidade genética das matrizes fornecedoras de sementes, para que sejam montadas estratégias de manejo e auxílio em áreas degradadas visando à conservação e manutenção da variabilidade genética de forma a assegurar gerações futuras.

Esta perda de recursos naturais tem sido consequência da ação antrópica, em que diversas espécies têm desaparecido, dentre elas a canafístula (Cassia grandis Linnaeus f.) e o pau-ferro (Caesalpinia ferrea var. leyostachia Benth), pertencentes à Família Leguminosa Caesalpinoideae. Ambas apresentam grande importância na vegetação marginal dos cursos de água, controlando a erosão, o assoreamento, auxiliando no controle da temperatura e também podem ser usadas como quebra-vento. $\mathrm{Na}$ área urbana, seu papel está relacionado a fins paisagísticos proporcionando bem-estar à população.

A importância do estudo da diversidade de espécies arbóreas, em programas de restauração dos ecossistemas degradados, vem se consolidando ao longo dos anos. Estes estudos promovem inferências quanto à escolha de indivíduos que funcionem como matrizes fornecedoras de sementes, assim como na forma de dispor as mudas dessas espécies no campo a fim de promover a sustentabilidade da futura população (Kageyama e Gandara, 2000). A canafístula e o pau-ferro são espécies pouco conhecidas geneticamente, e têm potencial uso em programas de restauração de áreas degradadas.

As análises da variação isoenzimática têm se expandido em pesquisas de genética florestal. As principais aplicações destas técnicas têm sido na investigação dos padrões de variação genética e dos sistemas de cruzamento. Estas aplicações aumentam o conhecimento da estrutura genética em populações naturais, aumentando a eficiência do programa e dos esforços de conservação de genes de árvores ameaçadas de extinção (Oliveira et al., 2002).
A conservação genética de uma espécie requer o conhecimento de seu sistema de reprodução, variabilidade e estrutura genética. Tais estudos podem ser eficientemente realizados com base em dados de marcadores bioquímicos, como isoenzimas (Furnier et al., 1986; Gillet \& Hattemer, 1989) Estes marcadores são importantes ferramentas para avaliar a estrutura genética e a distribuição da variabilidade entre indivíduos em programas de restauração.

Uma condição fundamental para que haja evolução é a existência de variação genética. A seleção natural atua entre as variantes dentro das populações em relação à adaptação ao ambiente, proporcionando variação entre populações e, por fim, variação entre espécies (Torggler et al., 1995). A conservação de espécies tem por base a manutenção dos níveis naturais de variabilidade genética nas populações (Yeeh et al., 1996).

$\mathrm{Na}$ região do Estado de Sergipe, as espécies arbóreas utilizadas para programas de restauração têm sido encontradas em número reduzido de indivíduos, em particular, para a espécie de pauferro e canafístula, somente foi possível encontrar cinco indivíduos na região do Campus da UFS no município de São Cristóvão, os quais têm sido empregados na coleta de sementes. Contudo, para que esta ação seja continuada, realizou-se o presente trabalho visando à verificação da proximidade genética destes cincos indivíduos de pau-ferro e canafístula utilizando marcadores enzimáticos. Com as informações de similaridade genética poderão ser definidas estratégias para coleta e conservação de sementes e obtenção de mudas, que serão empregadas para recuperação de áreas degradadas.

\section{MATERIAL E MÉTODOS}

\subsection{Material vegetal}

Folhas jovens de cinco indivíduos de canafístula e de cinco indivíduos de pau-ferro localizados no Campus da Universidade Federal de Sergipe (UFS), ambos fornecedores de sementes para programa de restauração de áreas degradadas, foram coletadas, sendo estes indivíduos georreferenciados com auxílio do aparelho GPS (Global Positioning System) - Etrex ${ }^{\circledR}$ modelo Camo. 


\subsection{Extração de enzimas}

Foram macerados dois gramas do material jovem e tenro de ambas as espécies, individualmente, com uso de almofariz e pistilo de porcelana previamente resfriados. Adicionou-se $1.000 \mu \mathrm{L}$ de Tampão $\mathrm{n}^{\circ} 1$ de Alfenas et al. (1991), $10 \mathrm{mg}$ de PVP e $10 \mu \mathrm{L}$ de $\beta$-mercaptoetanol. Após a maceração, os extratos foram colocados em microtubos de $1,5 \mathrm{~mL}$, previamente identificados e levados à centrifugação por 10 minutos a $4{ }^{\circ} \mathrm{C}$ e rotação de $12.000 \mathrm{RPM}$, sendo posteriormente armazenados em freezer a $-18{ }^{\circ} \mathrm{C}$, para que não ocorresse a degradação das enzimas.

\subsection{Corrida eletroforética}

Foram utilizados $30 \mu \mathrm{L}$ do sobrenadante em gel de poliacrilamida descontínuo, sendo o gel concentrador de $4,5 \%$ e o gel separador de $7,5 \%$ (Alfenas et al., 1991). A corrida vertical durou 3 horas e 30 minutos, sob amperagem de $10 \mathrm{~mA}$ à temperatura de $4{ }^{\circ} \mathrm{C}$.

\subsection{Revelação das enzimas}

Após a corrida eletroforética, procedeu-se à revelação dos géis segundo Alfenas et al. (1991), para o sistema enzimático Esterase (EST - 3.1.1.1), Glutamato Oxaloacetato Transaminase (GOT - 2.6.1.1), Peroxidase (EC - 1.11.1.7). Álcool Desidrogenase (ADH - EC 1.1.1.1) e Glucose Desidrogenase (GLUDH - EC 1.1.1.47). Após o surgimento das bandas, os géis foram retirados da solução de revelação, lavados em água corrente e fixados em solução de glicerol a 10\%.

\subsection{Cálculo da mobilidade relativa $(R F)$}

Foi empregada a fórmula sugerida por Alfenas (1991): $\mathrm{Rf}=(\mathrm{d} / \mathrm{D}) \times 100$, onde: $\mathrm{d}=$ distância percorrida pela molécula e $\mathrm{D}$ = distância percorrida pela corrente elétrica.

\subsection{Similaridade genética}

Foi utilizada uma matriz binária de 0 (ausência de banda) e 1 (presença de banda), realizando o cálculo de Similaridade Genética $\left(S_{i j}\right)$ entre os genótipos de cada espécie, de acordo com a fórmula proposta por Nei \& Li (1979) (Equação 1):

$\mathrm{S}_{\mathrm{ij}}=\frac{\mathrm{X}_{\mathrm{ni}} \times \mathrm{X}_{\mathrm{nj}}}{\mathrm{N}}$

em que: $\mathrm{S}_{\mathrm{ij}}=$ similaridade genética entre o genótipo i e o genótipo $\mathrm{j} ; \mathrm{X}_{\mathrm{ni}}=$ escore para a banda " $\mathrm{n}$ " no genótipo i; $\mathrm{X}_{\mathrm{nj}}=$ escore para a banda "n" no genótipo j; $\mathrm{N}=$ total de bandas avaliadas.

A matriz de similaridade genética obtida entre os genótipos foi submetida a uma análise pelo Método de Agrupamento pela Média (UPGMA) no programa NTSYS-PC (Rohlf, 2001).

\section{RESULTADOS E DISCUSSÃO}

Nas inferências sobre a diversidade genética de ambas as espécies, foram utilizados cinco sistemas enzimáticos, porém, apenas três apresentam atividade enzimática, alguns destes com locos altamente polimórficos sugerindo uma possível variabilidade genética. $\mathrm{O}$ pau-ferro apresentou maior número total de bandas e alelos do que a canafístula (Figura 1).

Para a espécie de pau-ferro, houve a presença total de 16 locos com 24 alelos; já para a canafístula,

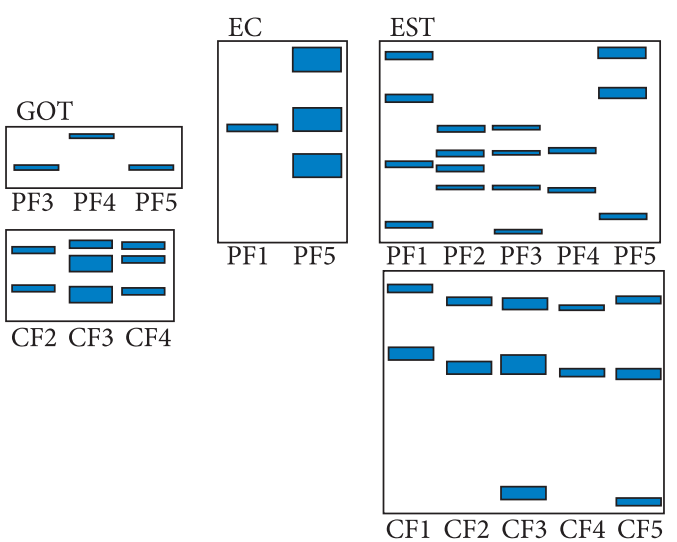

Figura 1. Zimograma obtido de três sistemas isoenzimáticos: Esterase (EST), Glutamato Oxaloacetato Transaminase (GOT) e Peroxidase (EC) para cinco indivíduos de Caesalpinia ferrea var. leyostachia Benth (PF) e Cassia grandis (CF).

Figure 1. Zimogrames obtained for tree isoenzimatic systems: Esterase (EST), Glutamate Oxaloacetate Transaminase (GOT) and Peroxise (EC) for five individuals of Caesalpinia ferrea var. leyostachia Benth $(\mathrm{PF})$ and Cassia grandis (CF). 
12 locos com 20 alelos, distribuídos entre os três perfis isoenzimáticos (Tabela 1).

Pinto \& Carvalho (2004) estudaram duas populações de Xylopia brasiliensis Sprengel por meio de sete sistemas isoenzimáticos, visando determinar os níveis de variabilidade genética mantidos entre e dentro das populações, verificaram a presença de 36 alelos totais distribuídos em 16 locos. No nosso estudo, pode-se inferir, em comparação com o trabalho anterior, que apesar do número reduzido de indivíduos e de sistemas utilizados, há um polimorfismo relativamente alto para as espécies.

O sistema EST foi o sistema que mais apresentou polimorfismo e locos para ambas as espécies, sendo todos polimórficos. Para a espécie de pau-ferro houve maior atividade enzimática, com 11 locos, e em canafístula 8 locos.

O sistema isoenzimático Esterase é constituído por um complexo e heterogêneo grupo de enzimas reativas com uma ampla gama de substratos específicos (Scandalios, 1969). As variantes dessas proteínas encontradas em plantas, por exemplo, são geralmente monoméricas ou diméricas (Weeden \& Wendel, 1990), com um alto nível de variabilidade (Gillespie \& Langley, 1974). Em razão disso, Esterase é um dos sistemas isoenzimáticos mais polimórficos em plantas (Weeden \& Wendel, 1990).

Para o sistema GOT, houve atividade para três das matrizes de pau-ferro (PF3, PF4 e PF5) com dois locos. Já para a canafístula, esse sistema apresentou maior quantidade de locos (quatro). As matrizes CF1 e CF5 não apresentaram atividade enzimática, e nas demais (CF2, CF3 e CF4) o número de bandas variaram de dois a três.

Resultado semelhante foi encontrado em espécies do mesmo gênero, o pau-brasil ( $C$. equinata), encontrando no sistema GOT somente uma zona de atividade enzimática, interpretada como um loco monomórfico com um alelo fixado (GiudiceNeto et al., 2004). A enzima Glutamato Oxalocetato Transaminase tem uma importante participação em reações de transaminação, durante a eliminação do nitrogênio dos aminoácidos e na formação de grupos "ceto" para o Ciclo de Krebs e gluconeogênese (Tanksley, 1983).

No sistema EC, somente a espécie de pau-ferro apresentou atividade enzimática, sendo que apenas para os indivíduos PF1 (uma banda) e PF5 (três bandas distintas), totalizando três locos.

O não aparecimento de banda no gel pode estar ligado a alelos nulos ou raros, ou ainda ocorre ocasião em que a segregação pode estar distorcida por modificações pós-transcricionais ou ainda por algum fator na hora da extração (Rick et al., 1979). Lima et al. (2002) complementa que a atividade da peroxidase pode ser alterada por fatores externos como luz ou outras radiações, estresse (sais e temperatura). Estas enzimas têm sido caracterizadas durante a germinação de sementes, assim como, nos estágios de crescimento (Egley et al., 1983; Van Huystee, 1987).

Observando a mobilidade relativa (Rf) entre os indivíduos, tanto para o pau-ferro quanto para a canafístula, nota-se que houve distintos valores para mobilidade relativa entre os perfis eletroferéticos adquiridos pelos sistemas enzimáticos (Tabela 2).

Para o sistema EST, para o pau-ferro, o maior valor da $\mathrm{Rf}$ foi observado para o indivíduo PF3 $(72,5)$ e a menor para PF4 $(24,36)$, já para a canafístula foram para o CF1 $(39,24)$ e CF5 $(78,75)$, respectivamente. Analisando a posição das bandas entre os indivíduos, percebe-se que os indivíduos

Tabela 1. Número total de locos e alelos para os sistemas isoenzimáticos: Esterase (EST), Glutamato Oxalacetato Transaminase (GOT) e Peroxidase (EC) para indivíduos de Caesalpinia ferrea var. leyostachia Benth (PF) e Cassia grandis $(\mathrm{CF})$.

Table 1. Total number of loco and alleles for isoenzyme systems: Esterase (EST), Glutamate Oxaloacetate Transaminase(GOT) and peroxidase (EC) to individuals of Caesalpinia ferrea var. leyostachia Benth (PF) and Cassia grandis (CF).

\begin{tabular}{|c|c|c|c|c|c|c|}
\hline \multirow{2}{*}{ Espécie } & \multicolumn{2}{|c|}{ GOT } & \multicolumn{2}{|c|}{ EST } & \multicolumn{2}{|c|}{ EC } \\
\hline & Locos & Alelos & Locos & Alelos & Locos & Alelos \\
\hline PF & 2 & 3 & 11 & 17 & 3 & 4 \\
\hline $\mathrm{CF}$ & 4 & 8 & 8 & 12 & - & - \\
\hline Total & 6 & 11 & 22 & 27 & 3 & 4 \\
\hline
\end{tabular}


Tabela 2. Mobilidade relativa dos sistemas isoenzimáticos: Esterase (EST), Glutamato Oxalacetato Transaminase (GOT) e Peroxidase (EC) para indivíduos de Caesalpinia ferrea var. leyostachia Benth (PF) e Cassia grandis (CF).

Table 2. Relative mobility the system isozymes: Esterase (EST), Glutamate Oxaloacetate Transaminase (GOT) and peroxidase (EC) to individuals of Caesalpinia ferrea var. leyostachia Benth (PF) and Cassia grandis (CF).

\begin{tabular}{|c|c|c|c|c|c|}
\hline \multirow{2}{*}{ Matrizes } & \multicolumn{2}{|c|}{ EST } & \multicolumn{2}{|c|}{ GOT } & \multirow{2}{*}{$\begin{array}{c}\text { EC } \\
\text { PF }\end{array}$} \\
\hline & PF & CF & PF & $\mathrm{CF}$ & \\
\hline \multirow{4}{*}{1} & $\mathrm{Rf}^{1}=25,00$ & $\mathrm{Rf}^{1}=39,24$ & - & - & $\mathrm{Rf}^{1}=18,28$ \\
\hline & $\mathrm{Rf}^{2}=33,75$ & $\mathrm{Rf}^{2}=50,63$ & & & \\
\hline & $\mathrm{Rf}^{3}=53,75$ & & & & \\
\hline & $\mathrm{Rf}^{4}=58,75$ & & & & \\
\hline \multirow{4}{*}{2} & $\mathrm{Rf}^{1}=43,75$ & $\mathrm{Rf}^{1}=40,00$ & - & $\mathrm{Rf}^{1}=41,77$ & - \\
\hline & $\mathrm{Rf}^{2}=51,25$ & $\mathrm{Rf}^{2}=51,25$ & & $\mathrm{Rf}^{2}=46,83$ & \\
\hline & $\mathrm{Rf}^{3}=55,00$ & & & & \\
\hline & $R f^{4}=60,00$ & & & & \\
\hline \multirow{4}{*}{3} & $\mathrm{Rf}^{1}=43,75$ & $\mathrm{Rf}^{1}=40,74$ & $\mathrm{Rf}^{1}=50,00$ & $\mathrm{Rf}^{1}=39,24$ & - \\
\hline & $\mathrm{Rf}^{2}=50,00$ & $\mathrm{Rf}^{2}=51,85$ & & $\mathrm{Rf}^{2}=43,03$ & \\
\hline & $\mathrm{Rf}^{3}=60,00$ & $\mathrm{Rf}^{3}=76,54$ & & $\mathrm{Rf}^{3}=49,36$ & \\
\hline & $R f^{4}=72,5$ & & & & \\
\hline \multirow{3}{*}{4} & $\mathrm{Rf}^{1}=53,16$ & $\mathrm{Rf}^{1}=41,25$ & $\mathrm{Rf}^{1}=46,15$ & $\mathrm{Rf}^{1}=39,24$ & - \\
\hline & $\mathrm{Rf}^{2}=62,02$ & $\mathrm{Rf}^{2}=53,75$ & & $\mathrm{Rf}^{2}=43,03$ & \\
\hline & $\mathrm{Rf}^{1}=24,36$ & & & $\mathrm{Rf}^{3}=49,36$ & \\
\hline \multirow{3}{*}{5} & $\mathrm{Rf}^{1}=34,61$ & $\mathrm{Rf}^{1}=42,50$ & $\mathrm{Rf}^{1}=50,00$ & - & $\mathrm{Rf}^{1}=7,53$ \\
\hline & $\mathrm{Rf}^{2}=70,51$ & $\mathrm{Rf}^{2}=53,75$ & & & $\mathrm{Rf}^{2}=18,28$ \\
\hline & & $\mathrm{Rf}^{3}=78,75$ & & & $R f^{3}=26,88$ \\
\hline
\end{tabular}

PF2 e CF3 (51,25), PF2 e PF3 (60,00) e CF4 e CF5 $(53,75)$ apresentaram a mesma mobilidade relativa. Para a o sistema GOT, os indivíduos de pau-ferro, o maior valor foi observado para PF3 e PF5 $(50,00)$ e o menor para PF4 $(46,15)$, já para a canafístula os valores foram verificados para o CF3 e CF4 (49,36; 39,24). Para o sistema isoenzimático EC, o maior e menor valor da $\mathrm{Rf}$ foi observado para o indivíduo PF5 $(26,88 ; 7,53)$ e houve entre PF1 e PF5 a mesma mobilidade relativa $(18,28)$. Entre todos os sistemas isoenzimáticos foram verificadas as mesmas $\mathrm{Rfs}$ entre o sistema EST e GOT (PF3 e PF5 - 50,00; PF31, PF3 e PF5 - 53,75)

As diferenças de mobilidades encontradas neste estudo contribuíram para avaliar o polimorfismo existente bem como a diversidade genética entre os indivíduos. A mobilidade relativa nas bandas no gel é importante para definir codominância, segregação de alelos, sendo bastante característico de enzimas monoméricas em que cada alelo de um loco tem uma banda caracterizada por uma distância de migração (Quirós, 1993). De acordo com Ballvé (1988), o deslocamento uniforme das bandas quanto à ocorrência de maior ou menor número de bandas em amostras extraídas em diferentes estádios de desenvolvimento são fenômenos comuns e refletem modificações pós-tradução e/ou expressão de locos específicos em certas fases de desenvolvimento do vegetal.

Autores concordam que a mobilidade relativa é importante para identificação de diferenças e similaridade entre espécie, além da identificação e caracterização de perfis de uma espécie (Lima \& Menezes, 2002). Os resultados de Rf apresentados permitem que estes sejam utilizados em outros trabalhos de investigação e empregados para comparação, pois não se encontram na literatura resultados deste tipo com estas espécies nativas.

Com base nos perfis eletroforéticos, foi possível obter o agrupamento entre os indivíduos a partir da similaridade genética de Nei \& Li (1979). Com base no dendrograma, utilizando o critério de corte $30 \%$ de similaridade genética para ambas as espécies, houve a formação de cinco grupos (Figura 2). Em canafístula, observou-se a formação de três grupos principais: o primeiro foi composto pelo genótipo CF1; o segundo por CF2, CF3 e CF4; e o terceiro grupo foi composto por CF5. As matrizes de pau- 


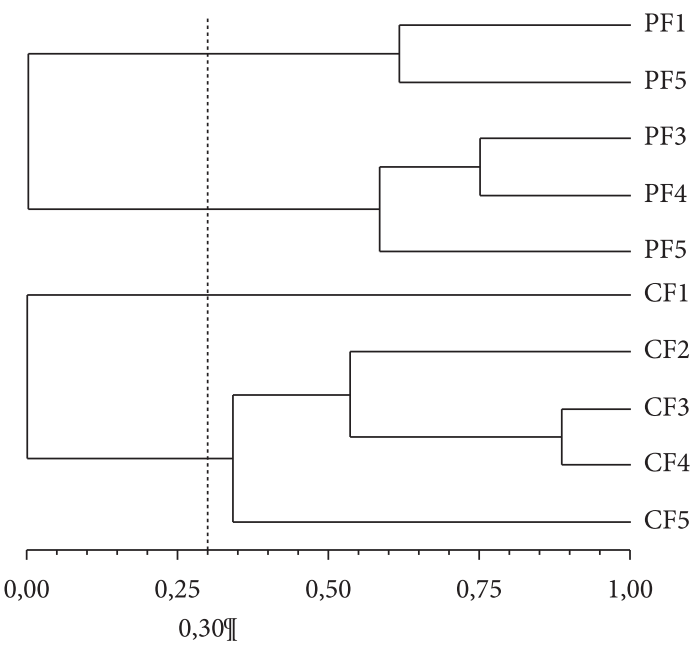

Figura 2. Dendrograma de similaridade gerado pelo método de agrupamento pela média (UPGMA) relativo aos indivíduos de Caesalpinia ferrea var. leyostachia Benth (PF) e Cassia grandis (CF), para Esterase (EST), Glutamato Oxaloacetato Transaminase (GOT) e Peroxidase (EC), agrupados de acordo com a similaridade de Nei \& Li (1979).

Figure 2. Similarity dendrogram generated by Unweighted Pair Group Method with Arithmetic (UPGMA) on individuals of Caesalpinia ferrea var. leyostachia Benth (PF) and Cassia grandis (CF) for Esterase (EST), Glutamate Oxaloacetate Transaminase (GOT) and Peroxise (EC), grouped according to similarity of Nei \& Li (1979).

ferro dividiram-se em dois grupos, sendo o primeiro formado pelos indivíduos PF1 e PF5, e o outro pelos indivíduos PF2, PF3 e PF4.

Os indivíduos de pau-ferro mais similares foram PF2 e PF3 (75\%), sendo o indivíduo mais divergente PF4 (59\%). Para canafístula, o par CF3 e CF4 (88\%) foram os mais semelhantes, ao contrário do indivíduo CF1 (100\%), que se destacou como o mais divergente. Com base nos resultados de similaridade genética, pode-se inferir que os indivíduos podem ser utilizados como matrizes fornecedoras de sementes para produção de mudas, contudo como há indivíduos semelhantes, como estratégia de coleta, a depender da época e da quantidade de indivíduos em frutificação, deve-se suprimir um dos indivíduos PF2 e PF3, para o pau-ferro, e CF3 e CF4, para a canafístula, a fim de promover a maior variabilidade genética entre as sementes que formarão as mudas que futuramente irão compor o ambiente degradado.

A técnica de isoenzimas auxilia na caracterização das distâncias genéticas, sendo o uso deste marcador útil para estimar diferenças entre indivíduos. Este tipo de estudo possibilita a obtenção de informações prévias da estrutura genética da população, permitindo, dessa forma, obter inferências sobre o nível de variabilidade e do potencial de manipulação, podendo os resultados gerados servir de ferramentas para definição de estratégias de conservação genética (Pimentel, 1988).

Assim, com base no exposto, as matrizes de pauferro e canafístula do Campus da UFS apresentaram variabilidade em relação ao padrão e número de bandas para os três sistemas enzimáticos, sendo as informações geradas úteis para originar informações para manejo das espécies em programas de restauração. São limitados os estudos sobre estas espécies em Sergipe, em especial o pau-ferro: uma espécie que apresenta caráter de risco de extinção no Estado e não é mais encontrada em algumas regiões.

\section{CONCLUSÕES}

O sistema Esterase apresenta maior polimorfismo e número de bandas para ambas as espécies.

Existe variabilidade genética entre os genótipos de C. ferrea var. leyostachia e C. grandis avaliados.

\section{STATUS DA SUBMISSÃO}

Recebido: 09/04/2009

\section{AUTOR(ES) PARA CORRESPONDÊNCIA}

\section{Renata Silva-Mann}

Universidade Federal de Sergipe - UFS, Av. Marechal Rondon, s/n, CEP 49100-000, Sao Cristóvão, SE, Brasil

e-mail: renatamann@gmail.com

\section{Allívia Rouse Ferreira dos Santos}

Universidade de Santiago de Compostela - USC, Campus Universitário de Lugo c/ Benigno Ledo, s/n, CP 27002, Lugo, Espanha e-mail: alliviarouse@hotmail.com 


\section{REFERENNCIAS}

Alfenas AC, Peters I, Brune W, Passador GC. Eletroforese de proteínas e isoenzimas de fungos e essências florestais. Viçosa: Universidade Federal de Viçosa; 1991.

Ballvé RML. Isoenzimas como marcadores genéticos em palmiteiro (Euterpe spp.) [dissertação]. Campinas: Universidade Estadual de Campinas; 1988.

Egley GH, Paul RN, Vaughn KC, Duke SO. Role of peroxidase in the development of water impermeable seed coats in Sida spinosa L. Planta 1983; 157:224-232. doi:10.1007/BF00405186

Furnier GR, Knowles P, Aleksiuk MA, Dancik BP. Inheritance and linkage of allozymes in seed tissues of whitebark pine. Canadian Journal of Genetics and Cytology 1986; 28:601-604.

Gillespie JH, Langley $\mathrm{CH}$. A general model to account for enzyme variation in natural populations. Genetics $1974 ; 76: 837-887$.

Gillet E, Hattemer, HH. Genetic analysis of isoenzyme phenotypes using single tree progenies. Heredity 1989; 63:135-141. doi:10.1038/hdy.1989.84

Giudice-Neto J, Sebbenn AM, Kageyama PY. Herança e ligação em locos isoenzimáticos de Caesalpinia echinata L. (pau-brasil). Revista do Instituto Florestal 2004; 16(2):101-110.

Kageyama P, Gandara FB. Recuperação de áreas ciliares. In Rodrigues RR, Leitão Filho, HF, editors. Matas ciliares: conservação e recuperação. 2. ed. São Paulo: EDUSP; 2000.

Lima GPP, Barsalobres C, Piza IMT, Cereda MP. Efeito do BAP e ANA e atividade da peroxidase em mandioca (Manihot esculenta Crantz Cv Mcol 22) cultivada in vitro. Revista Brasileira de Agrociência 2002; 8(2):107110.

Lima MLF, Menezes M. Estudo comparativo de isolados de Colletotrichum graminicola através da análise eletroforética de padrões protéicos e isoenzimáticos. Revista Fitopatologia Brasileira, 2002; 27(1):12-16.

Nei M, Li W. Mathematical model for studying genetic variation in terms of restriction endonucleases. In Proceedings of the National Academy of Sciences of the United States of America; 1979; Washington, DC. 76:5256-5273.
Oliveira AF, Carvalho D, Rosado SCS. Taxa de cruzamento e sistema reprodutivo de uma população natural de Copaifera langsdorffii Desf. na região de Lavras (MG) por meio de isoenzimas. Revista Brasileira de Botânica 2002; 25(3):331-338.

Pimentel AM. Utilização da técnica da eletroforese em genética florestal. Série Técnica IPEF 1988; 5(15):14-27.

Pinto SIC, Carvalho D. Estrutura genética de populações de pindaíba (Xylopia brasiliensis Sprengel) por isoenzimas. Revisat Brasileira de Botânica 2004; 27(3):597-605.

Quirós CF. Isoenzimas como marcadores genéticos para identificar híbridos en el cultivo de tejidos. In Roca WM, Mroginski LA., editors. Cultivo de tejidos en la agricultura, fundamentos y aplicaciones. Colombia: Centro Internacional de Agricultura Tropical - CIAT; 1993.

Rick CM, Tanksley SD, Fobes JF. A pseudoduplication in Lycospersicon esculentum. Proceedings of the National Academy of Sciences 1979; 76:3435-3439. doi:10.1073/ pnas.76.7.3435

Rohlf FJ. NTSYS-PC: numerical taxonomy and multivariate analysis system. New York: Exeter Software; 1992.

Scandalios JG. Genetic control of multiple molecular forms of enzymes in plants: a review. Biochemical Genetics 1969; 3:37-39. doi:10.1007/BF00485973

Tanksley SD. Isozymes. Part b. Amsterdam: ElSevier; 1983.

Torggler MGF, Contel EPB, Torggler SP. Isoenzimas: variabilidade genética em plantas. Ribeirão Preto: Sociedade Brasileira de Genética; 1995.

Van Huystee RB. Some molecular aspects of plant peroxidase biosynthetic studies. Annual Review of Plant Physiology 1987; 38:207-219.

Weeden NF, Wendel JF. Genetics of plant isozymes. In Soltis DE, Soltis PS, editors. Isozymes in plant biology. London: Chapman and Hall; 1990.

Yeeh Y, Kang SS, Chung MG. Evaluations of the natural monument populations of Camellia japonica (Theaceae) in Korea based on allozyme studies. Botanical Bulletin of Academia Sinica 1996; 37(2):141-146. 